\title{
Prevention of Gas Leakage Hazards in Home
}

\author{
Kokula Krishna Hari Kunasekaran1)
}

\begin{abstract}
The technology is growing faster in day to day life, and this technology is going to play a vital role in the lives of a human being. Almost all the people will use the gas-related items in their homes like the kitchen and other usages. The leaks for such gas leakages are a common problem, and due to this leakage, the severe health issues and other life death issues also can take place. Hence, the identification of such gas leakages is essential at the right interval of times. Having the learning essential to have the capacity to counteract and recognize a gas release right off the bat is critical with the end goal to avert potential gaseous petrol crises. Understanding what a flammable gas spill crisis is and how to respond is imperative to guarantee you and your family's security. In this paper, talking about what a gas spill crisis is and what to do if one happens, yet going to give you a wide-assortment of location and anticipation tips. Having this necessary information will limit you and your family's dangers for gas spills crises.
\end{abstract}

Keywords: LPG, Gas Sensor, Leakage Detection, Health Issues, Serious Problems, Methane, Carbon Monoxide, Thiophene, Mercaptan, Liquid Petroleum Gas

\section{Introduction}

When we are working with any gas related or gas-based units, it is always straightforward to operate and very highly useful to every human being. But, unfortunately, if any situation will arise due to the leakage of any gas pipes or any leakage from the sources or devices or units related with the gas-based units will be severe problems to the human beings. The problems arise maybe some sort of serious health issues, and in some cases, it will arise to more severe issues and sometimes the loss of human lives due to the leakage[1]. The most critical problems may be due to leakage was the carbon monoxide might be released from such gas leakage when it mixes with the natural air gases with the liquid petroleum gas with the gases available in the environment surrounded by it. When human beings and animals inhale this Carbon Monoxide in the environment, some serious health issues will be raised[2]. A minimal amount of gas leaks or very minute gas leaks may not have a significant impact or good damage as they may not have a significant impact and sometimes it is not possible to identify

Received(February 13, 2020), Review Result(1st: March 21, 2020, 2nd: May 18, 2020), Accepted(May 27, 2020)

1) (Association of Scientist) Developers and Faculties (ASDF), United Kingdom email: prm@kokulakrishnaharik.in 
such gas leakage also.

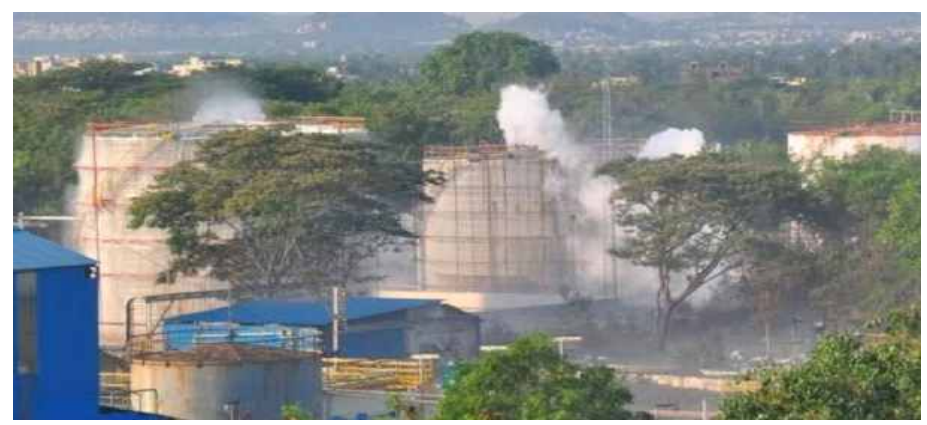

[Fig. 1] An Example of Gas Leakage Problems

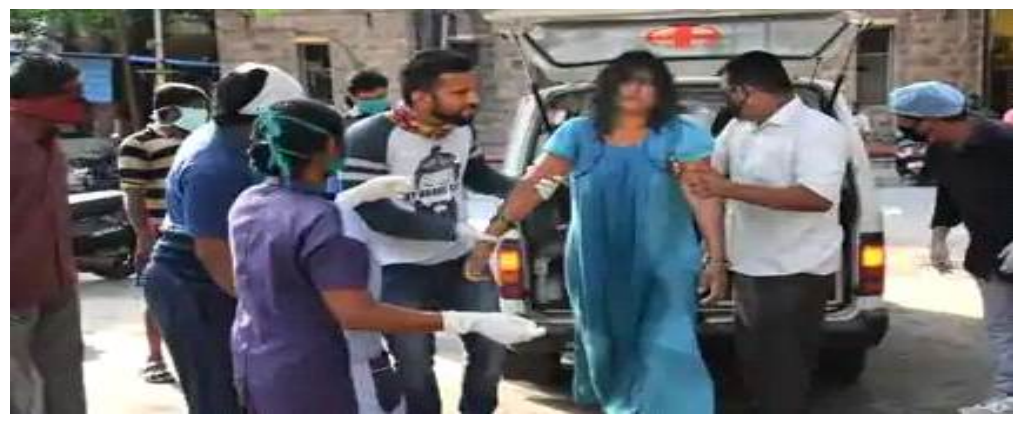

[Fig. 2] An Example of Woman being Suffering with Gas Leakage Problems

Some of the famous and severe things to be observed or identified for the leakage of gas from any source are like the sulfur smell, rotten eggs smell, some dust-like clouds forming near the gas line or some small sound like whistle coming out near the gas pipeline, observing the death of some indoor plants in and around the house also the damage of the gas pipes in and around the surroundings[3][4]. Some of the other symptoms observe din physical mode or any human being can observe such symptoms physically are like the breathing problems, getting difficult to breathe, dizziness observed on the face of the surrounding people, drowsiness, feeling drowsiness, chest pain, vomiting, vomiting sensation, reduced body weights etc. Whenever any human being is observing such symptoms, the immediate reaction and identification should be that there is a gas leakage in and around us in our premises. The necessary preventive steps to be taken to reduce the damage and also can save the lives of human beings and animals.

The most critical and pressing issue to be taken was that the carbon monoxide would be released or made available whenever the gas leakage had happened and when this gas leaked will mix with the surrounding environment and the other gases present in the environment. 
The emission of this sort of gas will leads to very serious issue sot human beings and the in and around surrounded human beings and animals. Severe issues will be available or can be observed in the human beings like the pain in the abdomen, massive pains in chest, body pains, headaches in dangerous levels, dizziness, burnings on hands, palms, skin etc[1][6].

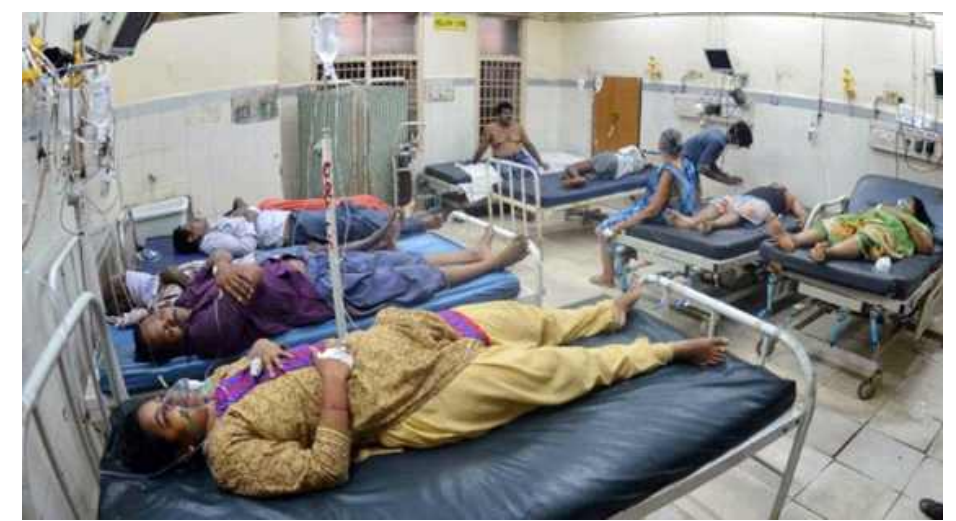

[Fig. 3] An Example of People being Suffering with Gas Leakage Problems[5]

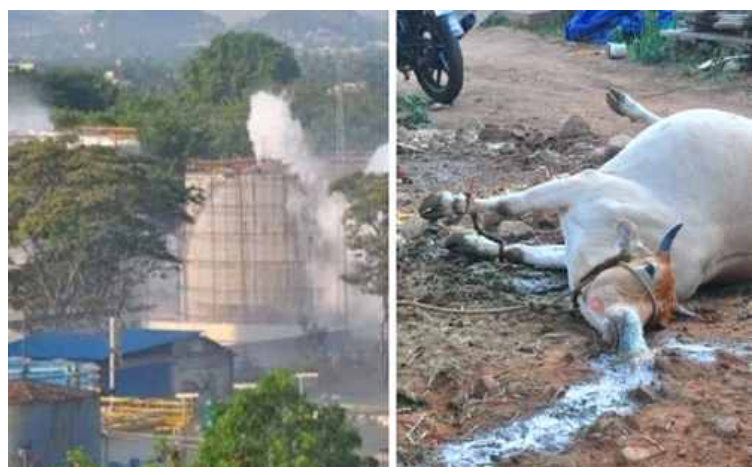

[Fig. 4] An Example of Animals being Suffering with Gas Leakage Problems[5]

The primary concerned or the severe problem that may cause these sorts of gas leakages are the respiratory problems. If the children and fewer age people are prone to such gas leakages, the effects or the damage may be more. Whenever there is a usage of any electronic gadget in the premises of gas leakages, the gas will be blown that may cause the death of several human beings. The utilization of several electronic gadgets and other fire letting devices should be banned.

LPG (Liquid Petroleum Gas) is generally utilized in homes for focal liquid petroleum gas warming, high temp water, gas-flames, cooking, and in versatile radiators for recreation exercises, for example, vessels, convoys and grills. The setbacks caused by this peril are as yet 
regular news in the media. Since the LPG in that capacity does not have any smell, gas organizations/refineries include an odorant, for example, ethanethiol, thiophene or a mercaptan so holes can be recognized effortlessly by the vast majority[1]. A remote home wellbeing gas spillage framework has been proposed in [3] where the alert gadget gives versatility inside the house premises.

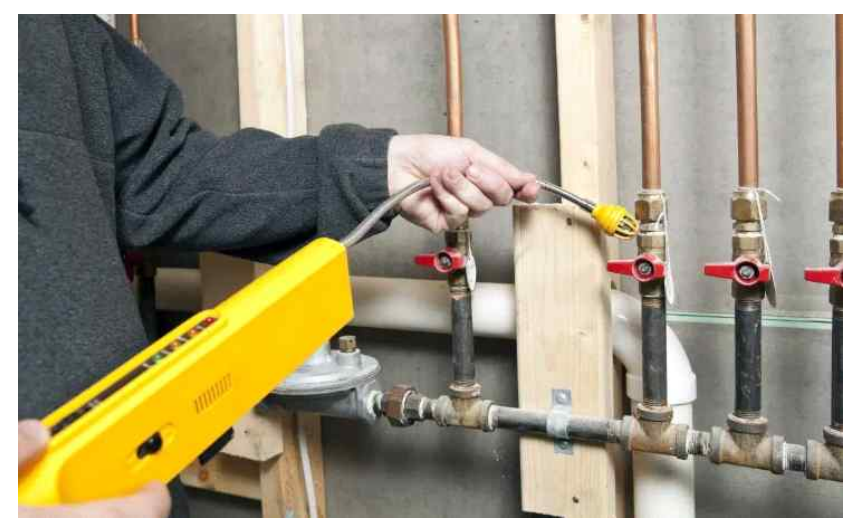

[Fig. 5] Gas Leakage Identification Process[5]

\section{Problem Formation}

Home flames have been occurring much of the time, and the danger to human lives and properties is developing as of late. Consequently, building up the gas spillage ready framework is exceptionally basic. Subsequently, this paper exhibits a gas spillage ready framework to recognize the gas spillage and to alert the general population locally available.

Distinguish Gas Leakage (like LPG spill, Butane spill, Methane spill) or any such oil-based vaporous substance that can be recognized utilizing MQ5 Sensor. Unfortunately, when you genuinely see a petroleum gas spill from the smell or sound, it has just turned into a potential gas crisis. That is the reason you must take deterrent measures to forestall spills before they happen.

\section{Prevention Methodology}

If you presume that there is a gas spill in your home, it is essential that you respond rapidly or else the outcomes can be extremely hindering. To begin with, leave your home promptly and make a point to not utilize any electrical gadgets, for example, light switches or phones. Power utilization could start or touch off the flammable gas spill. Open blazes could likewise 
cause this so make sure not to utilize matches or lighters. Finally, leave the zone and call your service organization to manage the issue. It is in every case best to give experienced experts a chance to fix any gas spills with the end goal to diminish the danger of gaseous petrol blasts. Try not to come back to the zone until the point when your home has been considered safe via prepared experts.

The following are the approaches for manual detection and prevention

1. SMELL the air. We add a particular scent to flammable gas with the goal that spills are less demanding to distinguish.

2. LISTEN for holes. A murmuring, shrieking or thundering sound close to a pipeline may show getting away petroleum gas. (Uncommon markers demonstrate the area of most significant pipelines.)

3. LOOK for pieces of information:

- A harmed association with a gas apparatus,

- Dead or kicking the bucket vegetation over or close to a pipeline

- A fire close to a pipeline

- Exposed pipeline after a seismic tremor, fire, surge or another debacle Working Mechanism.

The manual approach may lead to the time taken and less efficient due to human error. To minimize and improve the efficiency by the automated sensor-based system. Gas spills or smell can be detected using Sensor called MQ- sensor.

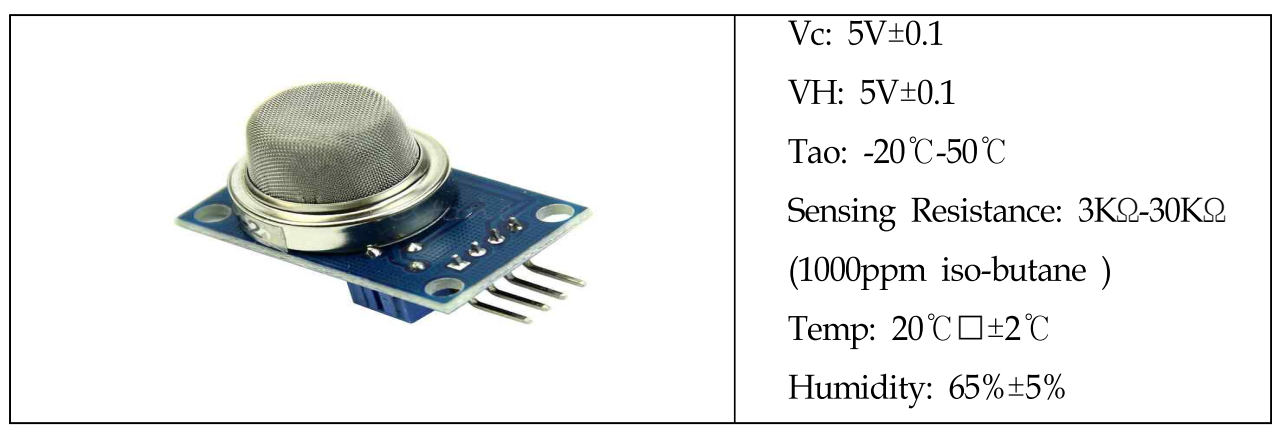

[Fig. 6] MQ 2 Sensor and Specifications

\subsection{MQ 2 Sensor}

The MQ-2 is a combustible gas, and smoke sensor recognizes the convergences of burnable gas noticeable all around and yields its perusing as a simple voltage. The Sensor can quantify groupings of combustible gas of 300 to $10,000 \mathrm{ppm}$. The MQ-2 gas sensor is delicate to LPG, 
I-butane, propane, methane, liquor, Hydrogen and smoke. They are utilized in gas spillage recognizing types of gear in family and industry and the compact gas locator.

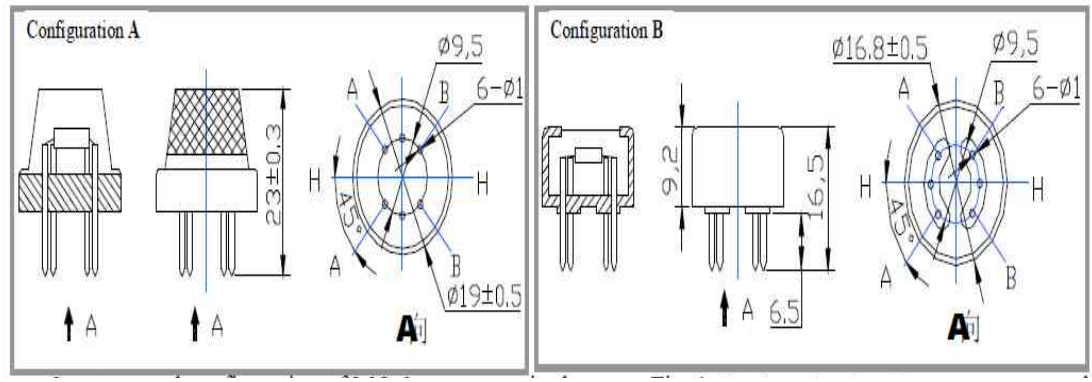

[Fig. 7] Working Mechanism of MQ2 Sensor

Structure and arrangement of MQ-2 gas sensor appear like [Fig. 1] (Configuration an or B), Sensor created by hull made by plastic and treated steel net. The radiator gives necessary work conditions to work of delicate segments. The wrapped MQ-2 has 6 stick, 4 of them are utilized to bring signals, and other 2 are utilized for giving warming current [3][7-12].

The resistance value of MQ-2 is the difference between various kinds and various concentration gases. So, when using this component, sensitivity adjustment is essential. We recommend that you calibrate the detector for $1000 \mathrm{ppm}$ liquefied petroleum gas $<\mathrm{LPG}>$ or 1000ppm iso-butane <i- C4H10>concentration in air and use-value of Load resistance that (RL) about $20 \mathrm{~K} \Omega(5 \mathrm{~K} \Omega$ to $47 \mathrm{~K} \Omega$ ). When accurately measuring, the proper alarm point for the gas detector should be determined after considering the temperature and humidity influence.

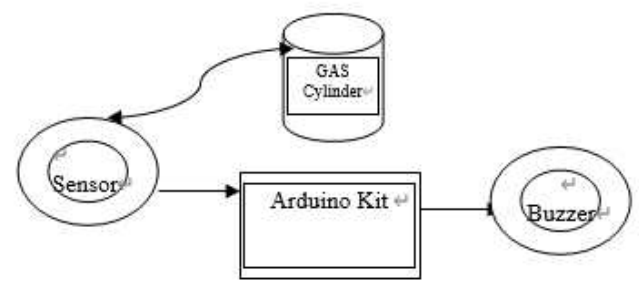

[Fig. 8] Model Block Diagram

\subsection{GAS Detection System}

The gas leakage model work flow diagram had given at [Fig. 9]. The total working process of the current model had given in the [Fig. 9] and also the physical model of the current system also displayed in [Fig. 10]. If any GAS leakage occurs, the system detects all the flammable 
gases. If the gas leakage crosses the Threshold it detects the gases like methane, butane, propane, LPG, and smoke. The entire unit will be controlled by a single Arduino board and all the other devices used in the model or kit can be controlled by this unit. Every piece of data being collected by these sensors and other units in the kit can be passed to the Arduino Unit and the decisions will be processed by this unit based on the information from sensor units. The unit will give the alarm if any leakage of gas will be observed in the premises or the range of the sensors used in the current model[13][14].

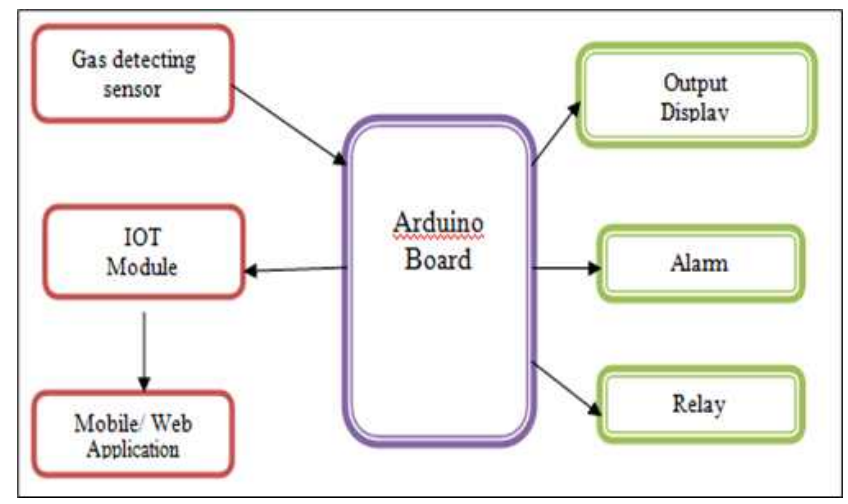

[Fig. 9] Working Flow of Gas Detecting System

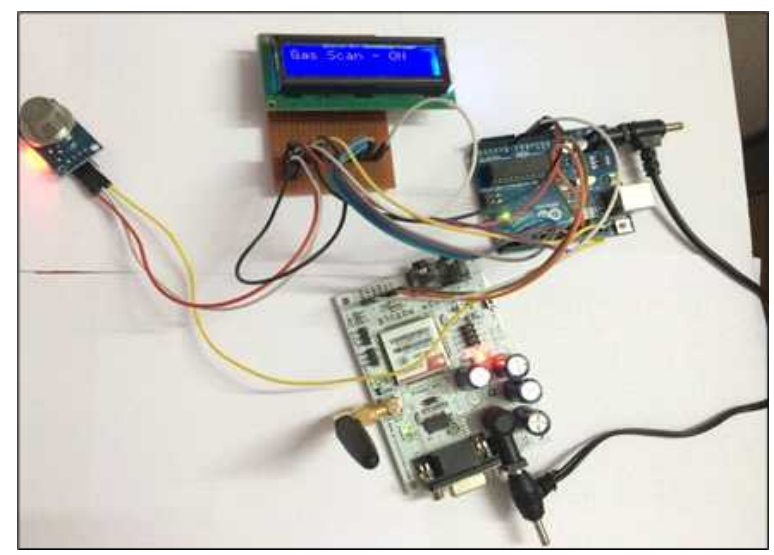

[Fig. 10] Experimental Setup

The actual physically developed model of the current gas leakage system had displayed in the above [Fig. 10]. All the sensors placed and used in the current system can be visually observed by the users from the [Fig. 10]. Upon detecting the gases, it turns $\mathrm{ON}$ the ventilator fans and sends an alert message to the user or the registered department. The system takes care of everything without a single input from the user. 


\section{Summary}

In the current article, gas leakage problems were highlighted, the effects of gas leakage on people also explained and discussed about the model of gas leakage detection. Gas spillage prompts extreme mischance bringing about material misfortunes and human wounds. Gas spillage happens chiefly because of poor upkeep of gear and lacking familiarity with the general population. Subsequently, LPG spillage identification is necessary to needed mishaps and to spare human lives. This paper disused about manual human based detection and how to over improve the performance using Sensor based mechanism. In future work, we need to develop a product with a lesser price and easy to operate by the average human.

\section{References}

[1] E. Jebamalar Leavline, D., Asir Antony Gnana Singh, B. Abinaya, H. Deepika, LPG gas leakage detection and alert system, International Journal of Electronics Engineering Research, (2017), Vol.9, No.7, pp.1095-1097.

[2] A. Mahalingam, R. T. Naayagi, N. E. Mastorakis, Design and implementation of an economic gas leakage detector, Proceedings of the 11th international conference on Applications of Electrical and Computer Engineering, (2012), March 7-9; Athens, Greece.

[3] Technical Data of MQ Sensor, HANWEI ELECTRONICS CO. LTD, https://www.hwsensor.com/

[4] D. Ganesh, A. Anilet Bala, Improvement on gas leakage detection and location system based on wireless sensor network, International Journal of Engineering Development and Research, (2015), Vol.3, No.2, pp.407-411.

[5] Leonard, How to recognize a gas leak, Medical News Today, J2018, https://www. medicalnewstoday.com/articles/321277.php\#prevention

[6] Pranay Meshram, IoT Based LPG Gas Leakage Detector, International Journal of Scientific Research in Computer Science, Engineering and Information Technology, (2019), Vol.5, No.1, pp.531-534.

[7] Min Goo Lee, Yong Kuk Park, Kyung Kwon Jung, Young Jin Kim, Evaluation of IR Gas Image System in Open Path Environments, International Journal of Advanced Science and Technology, (2015), Vol.83, pp.41-48, DOI: http://dx.doi.org/10.14257/ijast.2015.83.04

[8] Li Cheng, Liu Yan-ju, Zhang Hong-lie, An Improved Coal and Gas Outburst Prediction Algorithm Based on BP Neural Network, International Journal of Control and Automation, (2015), Vol.8, No.6, pp.169-176, DOI: http://dx.doi.org/10.14257/ijca.2015.8.6.17

[9] JooAh Lee, Mi-Hye Kim, Bong Hee Kang, Study on the Image Preference for the Development of the 
Vol.6, No.6, June 30 (2020), pp.121-129 http://dx.doi.org/10.21742/apjcri.2020.06.12

Application for the Gas Safety Field Workers, International Journal of Control and Automation, (2015), Vol.8, No.6, pp.211-220, DOI: http://dx.doi.org/10.14257/ijca.2015.8.6.21

[10] Kihwan Eom, Hanjoo Choi, Keehoon Won, Seoyeon Won, Kyung Kwon Jung, Temperature History System Using the NFC Self-powered Temperature Sensor Tag, International Journal of Smart Home, (2019), Vol.13, No.1, pp.7-14, DOI: 10.21742/IJSH.2019.13.1.02

[11] Jun-Hee Choi, Hyun-Sug Cho, A Study on the Improvement of the Accuracy of Low-Cost Light Scattering Method Particulate Matter Sensors, International Journal of Computer-aided Mechanical Design and Implementation, (2019), Vol.5, No.1, pp.1-8, DOI: 10.21742/IJCMDI.2019.5.1.01

[12] A. Manasa, Ch. Sudhakar, N. Thirupathi Rao, Attacks on Wireless Sensor Network: A Review, International Journal of Internet of Things and its Applications, (2019), Vol.3, No.1, pp.7-16, DOI: 10.21742/IJIoTA.2019.3.1.02

[13] Dinesh Sharma, Geetam Singh Tomar, A Survey on Energy Efficient Hierarchical Based Routing Protocols in Wireless Sensor Networks, International Journal of Wireless and Mobile Communication for Industrial Systems, (2019), Vol.6, No.1, pp.1-14, DOI: 10.21742/IJWMCIS.2019.6.1.01

[14] Seong-Se Cho, Seung-Hun Kim, Won-Hyuck Choi, Comparison of Communication Protocol Power Consumption of Fine-Dust IoT Sensor Based on one M2M Standard Protocol, International Journal of Internet of Things and its Applications, (2020), Vol.4, No.1, pp.7-14, DOI: 10.21742/IJoTA.2020.4.1.02 